\title{
Vom Ethos des Rezipienten
}

\author{
von Wolfgang Wunden
}

Während sich Kommunikationswissenschaftler und Ethiker nur langsam medienethischen Überlegungen zuwenden, haben Praktiker massenmedialer Kommunikation immer solche Probleme diskutiert. Sie sind ja ständig gezwungen, ihre Verantwortung wahrzunehmen und Entscheidungen plausibel zu machen: seien sie nun selber Kommunikatoren oder Kontrolleure von Kommunikatoren. Auch Kritiker von Programmentscheidungen - z.B. Presseredakteure im Bezug auf den Rundfunk - beteiligen sich an sehr praxisnahen Diskussionen über ethische Probleme, die bei Programmentscheidungen unterschiedlichster Art eine Rolle spielen. Um einige Beispiele zu nennen: ethische Argumentationen liegen der Diskussion über Gewaltdarstellungen im Fernsehen zugrunde; in der öffentlichen Diskussion über die Rolle der Massenmedien im Falle von Entführungen durch Terroristen nehmen sie einen breiten Raum ein: man diskutiert da die Frage, ob das Fernsehen die Forderung von Regierungen anerkennen soll, Informationen nicht zu publizieren, die es hat, um das Leben der entführten Personen zu schützen. Ethische Regeln stehen im Mittelpunkt der Erörterung in der Bundesrepublik Deutschland bezüglich der Rolle des Journalismus, insofern er in öffentlich-rechtlichen Institutionen praktiziert wird. Dieser Mangel an wissenschaftlichem Interesse ist äußerst bedauerlich.

\section{Ein weißer Fleck}

Der Mangel verschärft sich noch, wenn man feststellen muß, daß man sich im Bereich der Medienethik fast ausschließlich mit der Ethik des Kommunikators befaßt. Es fehlen ethische Aspekte der Struktur der Mediensysteme, welche auszudiskutieren so außerordentlich wichtig wäre, gerade jetzt, wo man - nicht nur in der Bundesrepublik - im Zuge der Einführung der neuen Technologien dabei ist, die bestehenden Strukturen schnell und nachhaltig zu verändern. Es fehlen aber auch - sieht man von ein paar Hinweisen von M. Schmolke, A. Auer und A. Huter ${ }^{1}$ ab Überlegungen zu einem Ethos des „Rezipienten“; Argumentationen sittlichen Gehalts also über das Verhalten des „Rezipienten“, über Werte, die er bei der Teilnahme an der Massenkommunikation verwirklichen sollte, über die Frage, welche Rechte und weiche Pflichten er dabei hat. Um diesen weißen Fleck auf der Landkarte medienethischer Überlegungen soll es in diesem Artikel vor allem gehen. Warum gibt es noch keine Rezipientenethik? Warum muß sie eine Kommunikatorenethik ergänzen?

Ein Versuch, die erste Frage zu beantworten, wäre interessant und würde eine detaillierte mediengeschichtliche Untersuchung voraussetzen. Aber dafür ist hier kein Platz. Zwei Gesichtspunkte sollen hier genannt werden, die Elemente einer Antwort darstellen könnten. So lange - erstens - Ethik als ein Versuch gelten mußte, in einer gegebenen Gesellschaft und ihrem strukturellen Rahmen sittlich verbindliche Regeln festzuschreiben, konnte sie nur Berufsethik sein, da die Gesellschaft ständisch zusammengesetzt war und von Königen und Fürsten regiert wurde.

Dr. Wolfgang Wunden ist Redakteur des Süddeutschen Rundfunks. 
Als im Verlauf des neunzehnten Jahrhunderts die Presse zu einem starken Stimulus der demokratischen Bewegung wurde und sich dementsprechend in Opposition zu den alten Kräften an der Regierung etablierte, als das Recht auf Information gegenüber jeder Form von Zensur öffentlich in Anspruch genommen wurde, da war die Zeit noch nicht reif dafür, die Leute, das Publikum an Pflichten zu erinnern, die sich auf den Gebrauch dieses Rechtes bezogen. Natürlich dachte jeder nur darüber nach, wie er die Grundfreiheiten gegen Eingriffe des Staates verteidigen könnte. Und im übrigen entspricht diese Denkweise der ältesten europäischen Tradition, beginnend mit dem Asylrecht. So mußte man bis zur jüngsten Zeit warten, etwa auf R. Marcic's Überlegungen zu neuen, aktiven Aspekten der Menschenwürde ${ }^{2}$, um das Recht auf Information in seiner umfassenden Qualität zu sehen, nämlich als ein Recht, das aktive Teilnahme am Leben von Gruppen innerhalb der Gesellschaft und am Leben der Gesellschaft im ganzen einschließt. „Menschenwürde” beschränkt sich eben nicht nur auf grundrechtlichen Schutz, sondern schließt die Verpflichtung des Einzelnen zu aktivem Vollzug dieser Würde ein.

Eine ähnliche Entwicklung vollzog sich - zweitens - in den Sozialwissenschaften, soweit die Rolle des „Rezipienten“ ihr Gegenstand ist. An sich hätten Theorien über den Prozeß der Massenkommunikation das Bild vom Teilnehmer daran verändern und erweitern können. Aber da die Verhaltensforschung in den Vereinigten Staaten Grundlage der Massenkommunikationsforschung war, entwarfen die Wissenschaftler Modelle der Massenkommunikation auf positivistische Art, d.h. Wirkungen von massenmedialen Botschaften wurden als „Antworten“ dargestellt, die durch Reize hervorgerufen und determiniert waren. Und als solche wurden sie Untersuchungsgegenstand. Die Rolle der Einzelperson, an die eine Botschaft gerichtet wurde, konnte höchstens als die eines mehr oder weniger passiven "Rezipienten" angesehen werden, der auf Reize reagierte, die vom Kommunikator her bei ihm ankamen. Soziologie und Sozialpsychologie erwiesen sich in diesem Fall als konservative, wenig zu gesellschaftlichen Entwicklungen anregende Wissenschaften.

Die augenblickliche Situation in der Massenkommunikation wird - trotz einiger Versuche, den „Rezipienten“ zu aktiver Teilnahme anzuregen - wohl korrekt im Bericht der „Internationalen Kommission für das Studium von Kommunikationsproblemen" (eine UNESCO-Studiengruppe unter dem Vorsitz von Sean MacBride) wiedergegeben: „... Trotz der je unterschiedlichen Bedingungen der Massenkommunikation ist der Mensch heute, und er wird es wahrscheinlich noch lange bleiben, weniger der Zentral-Handelnde und Meister in der Kommunikation, sondern viel mehr ein passives Ziel für Botschaften, ein Gegenstand der Kommunikation und ein großer Verbraucher von Informationsprodukten, bei deren Herstellung er nichts zu bestellen hat“ (Intereim Report \$95) : „... So wie die Praxis jetzt ist, ist das Individuum noch eher ein Objekt, das von professionellen Kommunikatoren gemanagt wird, als ein Subjekt, das als verantwortlicher Partner eigenen Rechtes handelt" (ebd., §94).

Unter diesen Umständen ist eine Ethik des „Rezipienten“ - nimmt man das Wort in dem Sinn, daß einer bloß aufnimmt, was andere ihm anbieten - per definitionemunvorstellbar. Ethik kann ja nur einen Sinn haben, wo Personen handeln, die verantwortlich sind für etwas, was sie tun, oder für etwas, was in einer Situation oder in einem Prozeß sich ereignet, zu der bzw. zu dem sie durch ihre Haltung und ihre 
Handlungen beitragen. So setzt eine Ethik des Rezipienten eine kopernikanische Wende voraus. In der Tat muß dann eine neue Ära in der Betrachtungsweise des Prozesses der Massenkommunikation eröffnet werden: der „Rezipient“ darf dann nicht länger die Rolle spielen, wie sie im MacBride-Report beschrieben ist; er muß vielmehr eine aktive Rolle spielen, bei der dann ethische Aspekte mitgedacht sind.

Warum braucht es solch eine kopernikanische Wende? Warum brauchen wir eine Ethik des Rezipienten? Die Entwicklung westlicher Gesellschaften mit ihrer Vorherrschaft wirtschaftlicher Macht tendiert dahin, die Identität der Individuen und die darauf beruhende Kommunikation zwischen ihnen zu reduzieren. Humane Werte werden mehr und mehr den Ansprüchen ökonomischer Planung geopfert. Sie eröffnen auf der einen Seite neue Techniken im Feld der elektronischen Kommunikation (Kabelfernsehen und Satelliten), neue Märkte für die elektronische Industrie und Arbeitsplätze für viele Menschen, auf der anderen Seite bringen sie wohl kaum mehr demokratische Kommunikation und mehr Partizipation zustande. Das Kabelfernsehen in den Vereinigten Staaten hat nicht zu mehr Qualität im Fernsehen geführt, hat die Hoffnungen nicht erfüllt, wie sie die SloanKommission in ihrem Bericht aus dem Jahr 1972 formulierte, daß nämlich das $\mathrm{Pu}$ blikum sich in stärkerem Maße an den Programmen des Kabelfernsehens beteiligen würde. Der Fehlschlag des Konzepts der amerikanischen FCC im Bezug auf public-access-Programme mag als Beleg für die allgemeine Regel gelten, daß technische Innovationen nicht von sich aus die Kommunikation verbessern, wenn sie nicht unter mehr als technischen und ökonomischen Gesichtspunkten gewertet werden.

Wenn die Kommunikation zwischen Menschen - und darin ist eingeschlossen die Kommunikation mittels Massenmedien - sich verbessern soll, wenn die Identität von Menschen gewahrt und gestärkt werden soll unter den Bedingungen moderner Massenkommunikation, brauchen wir eine Rezipientenethik, die der Mentalität reiner Verbraucher von Programmen, Botschaften und Meinungen entgegenwirkt. Wenn man die inneren Kräfte des Menschen nicht stärkt, kann eine Verbesserung humaner Kommunikation nicht erreicht werden. Dazu gehört sehr vieles, unter anderem auch die Entwicklung einer Rezipientenethik. Sie kann dazu helfen, immer mehr Leute zu sensibilisieren und das Publikum der modernen Massenmedien bewußt werden zu lassen, was es mit demokratischer Kommunikation und seiner Rolle darin auf sich hat.

\section{Ein neues Elhos}

Bis hierhin wurde schon deutlich, daß es uns bei einer Rezipientenethik nicht in erster Linie darum geht, was der Rezipient für Rechte hat, die auf Seiten der Journalisten oder Institutionen der Massenkommunikation entsprechende Pflichten mit sich bringen. Sie sollten hier aber wenigstens erwähnt werden, verbunden mit dem Hinweis auf Artikel 19 der Charta der Vereinten Nationen, in dem das Recht auf Information zugrundegelegt worden ist. Es geht uns viel mehr um ein neues "Ethos" der Personen, die das Publikum der Massenkommunikation ausmachen: d.h. die Leser, Hörer und Zuschauer, die wir gegen unsere Überzeugung

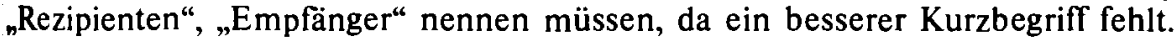
Nicht nur der Kommunikator, sondern auch der Rezipient ist dafür verantwortlich, 
daß Kommunikation gelingt. Er ist dafür nicht nur verantwortlich im Bereich primärer Kommunikation und in primären sozialen Beziehungen, sondern auch im Bereich der Massenkommunikation, was nicht so geläufig ist. Einige grundlegende Themen eines Rezipientenethos wurden von deutschen Philosophen und Ethikern notiert. R. Guardini z.B. wies auf einen wesentlichen Punkt hin, nämlich die innere Beziehung zwischen der Integrität der Privatsphäre und funktionierender öffentlicher Kommunikation ${ }^{3}$. Die letztere setzt die erstere voraus. Aus Guardinis Gedanken muß man folgern, daß deswegen nicht nur auf Seiten des Kommunikators eine sittliche Pflicht besteht, die Privatsphäre der Menschen zu respektieren, sondern auch eine sittliche Pflicht auf Seiten des einzelnen Menschen, nicht ohne Not seine eigene Privat- und Intimsphäre zum Gegenstand öffentlicher Berichterstattung zu machen. Verantwortung für das Gelingen von Kommunikation meint in diesem Zusammenhang, das man die eigene Subjekt-Qualität gegen die eigenen Tendenzen in Schutz zu nehmen hat, den inneren Bereich freiheitlicher Selbstverfügung zu öffnen und ihn so zu zersetzen und zu zerstören.

Dieses Beispiel zeigt, daß das Ethos des Rezipienten nicht ein Sonderethos ist, das sich nur auf die Rolle „Rezipient“ bezieht, sondern daß es sich auf die wesentlichen Qualitäten freier Individuen, freier Subjekte als solcher bezieht und sich nur auf die Bedingungen der Massenkommunikation hin artikulieren und spezifizieren muß. Das Ethos des Rezipienten - Ethos jetzt unterschieden von einer Ethik als einem Komplex sittlicher Regeln - ist also Teil einer allgemeinen Lebens-ordnung, die sich der einzelne nach den Kriterien praktischer Vernunft gibt. A. Auer hat einige Grundelemente eines solchen Ethos skizziert ${ }^{4}$. Ein solches Ethos verlangt zunächst eine Grundentscheidung für Spontaneität. Gegen jede Art von Konformismus sucht der spontane Mensch in den Medien, was ihm in seiner Lebensausrichtung weiter hilft, er wird es bewußt auswählen und es in personaler Weise verarbeiten. - Hinwendung zur Realität ist der von Auer an zweiter Stelle genannte Teil eines Rezipientenethos. Im Lebensprozeß muß jeder zusehen, daß er von Idolen und Illusionen wegkommt, um das Leben, so wie es ist, als Medium zu erkennen und anzunehmen, in dem er seine Identität finden kann. Wer das gelernt hat, der wird nicht mehr nur das spektakuläre Ereignis, die aufwühlende Geschichte in den Medien suchen. - Drittes Element eines Rezipientenethos: der Versuch, die eigene Freiheit und Verantwortung wahrzunehmen. Dies als Gegengewicht gegen Manipulationen und einseitige Darstellungen seitens der Medien. - Wille zur Ganzheit als Gegengewicht gegen partielle Darstellungsweisen der Medien, Wille zum Sinn als Gegengewicht gegen Frustrationen und Deprivationen, wie sie in den Medien breiten Raum einnehmen, und tolerante Lebenseinstellung, die sich darin beweist, daß man es ertragen kann, wenn Meinungen geäußert werden, die den eigenen entgegengesetzt sind: dies sind weitere Elemente in Auers Darstellung eines Ethos des Rezipienten. Schließlich und endlich gehört für ihn dazu der Wille, sich in primären Gruppen und elementaren sozialen Gebilden zu beheimaten, damit man einen sozialen Standort einnimmt, der einen befähigt, so vielen verschiedenen Meinungen und gegensätzlichen Informationen mit einiger Sicherheit gegenüberzutreten.

Diese Grundeinstellungen gegenüber den Massenmedien müssen sicherlich ergänzt werden durch andere Elemente, die sich spezifischer auf die Rolle „Rezipient" beziehen. Traditionelle christliche Medienethik hat die Pflicht des Rezipienten herausgestellt, z.B. „schlechte“ Zeitungen und Illustrierte zu meiden, 
solche Filme nicht zu sehen, um Schäden für die Seele zu vermeiden, wie M. Schmolke anmerkte 5 . Die Pastoralinstruktion „Communio et Progressio" hat einige konkrete sittliche Pflichten genannt, die zur Rezipienten-Rolle gehören: Z.B. die Pflicht, danach zu streben, informiert zu sein, als Pendant zum Recht auf Information. Oder die Pflicht, die Situation des Journalisten richtig einzuschätzen und sich selber aus verschiedenen Quellen zu informieren. Schließlich sollte der Rezipient sich über die Medien als solche informieren. Papst Paul VI. schließlich hat die Verantwortung für die Medien insgesamt genannt, die der Rezipient durch seine Auswahl von Medien bzw. Programmen wahrnimmt („Abstimmung am Kiosk"). M. Schmolke hat diese katholischen Stimmen zitiert und glaubt, daß es die wichtigste Pflicht des Rezipienten ist, sich seiner Rolle als Rezipient bewußt zu werden. Ohne die Partnerschaft des Rezipienten können weder privat- noch öffentlich-organisierte Medien sinnvoll funktionieren. Man muß aber auch etwas über die Medien und ihre Struktur wissen, die Medien sind ja Instrumente, vermitteln Inhalte in bestimmten Zusammenhängen; sie sind keinesfalls problemlose Unterhalter für den Abend.

Einige praktische Erfahrungen aus der Welt des Rundfunks mögen schließlich unterstreichen, wie wichtig ethische Elemente in der Rolle des Rezipienten und in der Funktionsweise der Medien sind.

Das erste Beispiel bezieht sich auf das, was die zitierte vatikanische Erklärung das Verständnis für die Situation der Journalisten nennt. G. Frei, der für ungefähr 20 Jahre Direktor des Schweizer Fernsehens war, schrieb anläßlich seines Ausscheidens aus dieser Funktion folgendes: „Was mir Sorge bereitet, ist die wachsende Ignoranz und, damit verbunden, die Intoleranz gegenüber Wesen und Funktion des Journalisten und Programmschaffenden in unserer freien demokratischen Gesellschaft, wie sie bei den Trägern der politischen Öffentlichkeit, den Parteien, Behörden, Verbänden, Institutionen, immer deutlicher spürbar wird. Es scheint immer schwieriger zu werden, die Einsicht zu bejahen, daß der Journalist seinem beruflichen Auftrag gemäß Partei nimmt, für das Neue, das Künftige, das, was nicht im Lot ist; daß er ex offizio sein Mißtrauen zum Ausdruck bringen muß gegenüber jeder Form von Macht (. . .). Und es scheint Mühe zu bereiten, zu verstehen, daß der Journalist - jenseits von gängigen Mehrheitsverhältnissen - immer wieder seine Präferenz zum Ausdruck bringt für das Unbeachtete, das Seltene, das Ungewöhnliche, das Minoritäre, und sich deshalb immer wieder - oft unter bewußter Mißachtung der Gesetze der Verhältnismäßigkeit - einsetzt für Gruppen unserer Gesellschaft, die keinerlei Macht haben, aber vielleicht ein paar stichhaltige Argumente. ${ }^{\prime 6}$

Sehr oft mißversteht das Publikum die Rolle der Medien und der Journalisten in einer freien Gesellschaft. Statt daß die Leute die Medien als Markt von Informationen und Meinungen verstehen, erwarten sie vom Medium Informationen und Meinungen, die mit ihren eigenen übereinstimmen und sie bestätigen. Diese Tendenz, eigene Bedürfnisse und Vorstellungen zum Kriterium für das zu machen, was Medien und Journalisten bieten sollen, ist weit verbreitet im Publikum, und dies führt oft dazu, daß Leute Medien und Journalisten verurteilen.

Ein zweites Beispiel weist auf die Rolle hin, die der Rezipient in der Kontrolle des öffentlichen Rundfunks spielen kann. Im öffentlich-rechtlich organisierten Rundfunk der Bundesrepublik Deutschland üben Rundfunkräte die Kontrollfunk- 
tion aus. Sie sind zwar verschieden zusammengesetzt je nach dem, wie das Gesetz für die einzelnen Anstalten es festlegt, doch haben sie prinzipiell dieselbe Funktion: sie sollen mittels der sogenanten ,relevanten“ Gruppen der Gesellschaft das Publikum repräsentieren. Fragt man nun den Durchschnittszuschauer oder den Durchschnittshörer, durch wen er im Rundfunkrat repräsentiert ist, bekommt man kaum einmal eine Antwort. Dies zeigt, daß nahezu niemand weiß, auf welchem Wege er die Macht, die er als Rezipient hat, ausüben kann, mag sie noch so minimal sein.

Ein drittes Beispiel bezieht sich - was die Bundesrepublik angeht - auf die Zukunft. Bis jetzt sind die Möglichkeiten des Rezipienten, seine passive Rolle als Rezipient in eine aktive Rolle im Kommunikationsproze $B$ zu verwandeln, sehr gering. Kabelfernsehen wird größere Möglichkeiten dazu eröffnen, und es scheint, daß die Regierungen bereit wären, finanziell zu helfen, daß einzelne Personen und Gruppen einen Fernsehkanal dazu benützen, ihre Meinungen und Probleme darzustellen und ein größeres Publikum damit zu konfrontieren. Aber nach den Erfahrungen in den Vereinigten Staaten muß man befürchten, daß die Chancen solcher „offener Kanäle“ im deutschen künftigen Kabelfernsehen nicht groß sind. Der ethische Wert, Massenkommunikation durch Beiträge des „Ex-Rezipienten“ zu verbessern, scheint noch unentdeckt zu sein und kein ausreichendes Echo zu finden. Nebenbei: manche Leute warten darauf, daß solch ein Experiment von vornherein scheitert, um dann sagen zu können: alles kann so bleiben wie es ist.

\section{Der kompetente Rezipient}

Es gibt keine Pädagogik ohne Werte oder Ideale, auf die hin der Pädagoge die Menschen zu erziehen versucht. Nun haben seit dem Aufkommen der Massenmedien Versuche stattgefunden, Regeln für den Gebrauch der Massenmedien zu lehren und zu vermitteln. In der aktuellen Diskussion über die Einführung neuer elektronischer Techniken hat die Diskussion über eine wirksame Medienpädagogik eine größere Aufmerksamkeit gewinnen können. Für viele, die die Einführung des Kabelfernsehens wünschen, dient der öffentlliche Ruf nach solch einer Pädagogik als Alibi für wirtschaftliche Interessen. Andere rufen nach Argumenten aus der Medienpädagogik mit dem Ziel, die Einführung des Kabelfernsehens zu verhindern. Beide Seiten berufen sich auf das Konzept des „mündigen“ Rezipienten, d.h. des Lesers, Hörers und Sehzuschauers, der in der Lage ist, die Medien rational und maßvoll zu gebrauchen, ohne daß dem quantitativen und qualitativen Output der Medien irgendwelche staatlich verordneten Grenzen gesetzt wären. Die einen setzen voraus, daß der Rezipient schon mündig ist, während die anderen voraussetzen, daß er es noch nicht ist. G. Maletzke, der diesen Begriff diskutiert, der eine gewisse Bedeutung für die aktuelle medienpolitische Debatte in der Bundesrepublik hat, erklärt in einem kurzen Aufsatz ${ }^{7}$, wie der mündige Zuschauer das Fernsehen gebraucht. Die Medienforschung hat herausgefunden, daß die Zuschauer das Fernsehen tatsächlich als Unterhaltungsinstrument ansehen, als ein Mittel für Entspannung und Ablenkung in der Freizeit. Informationssendungen werden weniger angesehen. Und das Ausmaß, in dem sie angeschaut werden, hängt sehr davon ab, was in den Kanälen geboten wird. Dies ist ein Grund, warum Kritiker der modernen Massenmediengesellschaft nach der „Ambulanz“ einer Medienpädagogik rufen. 
Für sie ist der „mündige“ Zuschauer nur eine Ausnahme: für die Masse des Publikums sollte er eine Soll-Vorstellung werden, an die die Pädagogen sie heranführen sollten. G. Maletzke schlägt vor: das Konzept des „mündigen“ Rezipienten aufzugeben, da es von Medienpolitikern mißbraucht werde und zur Verwirrung führe. Wer diesem Rat folgt, kann deswegen doch nicht einem Konzept oder einem Wert als allgemeinem Ziel für pädagogische Arbeit entraten. In Deutschland hat D. Baacke das Konzept der „Kompetenz“, das aus der Linguistik und aus Studien zur Sozialmedizin stammt - in den Bereich der Medienpädagogik eingeführt ${ }^{8}$. Dieser Begriff meint Selbstbestimmung und Autonomie in der Kommunikation, aktive Beteiligung am Gespräch der Gesellschaft und Definition sozialer Wirklichkeit mittels sprachlichen Handelns. Die Vorstellung der Kompetenz setzt ein Individuum in der Gesellschaft voraus, das gelernt hat, seine Kommunikationssituation in einem doppelten Sinn zu verstehen: Es versteht seine eigene innere Welt und deren Erfahrungsraum als Ursprung und inhaltliche Grundbedingung für seine Kommunikation mit der Umwelt, und es vermag mit der Wirklichkeit außer sich umzugehen, sei es auf dem Weg unmittelbarer Erfahrung oder auf dem Weg über Medienerfahrung.

Ein wesentliches Element von Medienkompetenz besteht wohl auch darin, daß der Rezipient in der Lage ist, sein eigenes sittliches Wertgefühl und seine sittlichen Maßstäbe mit den Wertüberzeugungen zu vergleichen, die über die Medien an ihn herangetragen werden. Insbesondere bei solchen Themen, die einen offensichtlichen sittlichen Gehalt haben (Fragen aus dem Sexualbereich und Fragen politischer Moral), gibt es häufig Unterschiede zwischen dem, was die Leute denken, und dem, was Autoren und Redakteure denken und äußern. Im Rahmen von Medienpädagogik hat die Medienethik hier viel zu tun: der „mündige“ Zuschauer, Hörer und Leser soll sich auseinandersetzen mit Werten, welche Medien an ihn heranbringen, und sich dabei seiner eigenen Wertüberzeugungen vergewissern; dabei soll er auch die spezifische Funktion der Medien in der sozialen Kommunikation respektieren, wozu nicht nur die soziale Integration gehört, sondern auch die Förderung menschlicher Werte und Belange von Minderheiten. Medienethik hat auch nicht offen geäußerte, sondern ,verschlüsselte“ Wertvorstellungen zu analysieren und ihre Wirkungen auf den Rezipienten abzuschätzen. In der Bundesrepublik gibt es Bemühungen, hier zu einigen Kriterien und Übungsmodellen zu kommen; jedoch sind - wie eingangs erläutert - auf dem Weg zu einer Ethik des Rezipienten erst einige wenige Schritte getan.

\section{Anmerkungen}

Dieser Artikel ist die leicht gekürzte Fassung eines Beitrags zu einem Sammelband in englischer Sprache. A. van der Meiden: Ethics and Mass Communication, Utrecht 1980.

1 Michael Schmolke: Rezipienten ohne Bewußtsein? in: „Communicatio Socialis“, Paderborn, 10:1977, S. 31-39: - Alfons Auer, Verantwortete Vermittlung - Bausteine einer medialen Ethik, in: Zentralstelle Medien der Deutschen Bischofskonferenz und Katholische Akademie Stuttgart (Hrsg.): Telekommunikation - ein Fortschritt für den Menschen? Stuttgart 1979, S. 61-80. - Alois Huter, Mensch und Massenmedien. Anthropologische Anmerkungen zur Medienforschung als Voraussetzung für eine Ethik der Massenmedien. Unveröffentlichtes Manuskript. Salzburg 1979. 
René Marcic: Ein neuer Aspekt der Menschenwürde, in: Staat Recht Kultur. Festgabe für E. Hippel. Bonn 1965, S. 189-203.

3 Romano Guardini: Freiheit. Eine Gedenkrede. Würzburg 1960, S. 20-23.

Gerhard Maletzke: Mündige Bürger - mündige Zuschauer? in: „Südfunk“, Stuttgart, 12 : 1979/12, S. 3-4.

8 Dieter Baacke: Kommunikation und Kompetenz. München ${ }^{2} 1975$.

9 Vgl. Wolfgang Wunden: Medienpädagogik - Führerschein fürs Fernsehen? Stuttgart 1980. Besonders S. 14-19.

\section{SUMMARY}

So far, neither communications scientists nor specialists in ethics have given sufficient thought to the ethical questions which for some time have engaged the attention of communications practitioners. Some fields of responsible journalism have been examined professionally, but moral and ethical aspects affecting the recipient, who has an important role in the communications process, have received little or no attention. Leaving aside theoretical questions, some basic values are given, the realisation of which by the recipient would help towards successful communications. Finally, the importance of these values for the development of media education is dealt with, and this should contribute towards a competent use of mass media.

\section{RÉSUMÉ}

$\mathrm{Ni}$ les chercheurs en matière de communication, ni les moralistes ne se sont, jusqu'à présent, occupés des termes éthiques du problème des medias, dans une aussi grande mesure que les communicateurs et les responsables du domaine des medias n'ont eu à le faire depuis toujours. Alors que les problèmes d'un journalisme responsable étaient toujours l'objet de travaux scientifiques, les réflexions quant à une éthique du receveur qui soit digne de son rôle actif dans le processus de la communication sont entièrement absentes. Quelques valeurs fondamentales, dont la réalisation par le receveur peut contribuer à la réussite de la communication des mass media, sont nommées sans que pour cela soient développés des fondements théoriques. Finalement, on esquisse la signification de telles représentations de valeurs pour le développement d'une pédagogie qui doit amener à un usage compétent des mass media.

\section{RESUMEN}

Ni los especialistas en medios de comunicación ni los moralistas se ocuparon hasta ahora suficientemente de la formulación de los problemas éticos de la comunicación, tal como deberían haber hecho tanto los teóricos de la comunicación social como los responsables en este sector. Aunque los problemas de un periodismo responsable fueron ya objeto de estudios científicos, se carece, por el contrario, de consideraciones sobre una ética del sujeto receptor en relación con su papel activo en el proceso de la comunicación social. Sin desarrollar una base teórica, se citan algunos valores básicos cuya realización por los receptores puede contribuir a que tenga eficacia la comunicación social. Por último se describe la importancia de tales conceptos de valor para el desarrollo de una pedagogía que debe conducir a una relación competente con los medios de comunicación social. 